\title{
Altered cortical development in fetuses with isolated non-severe ventriculomegaly assessed by neurosonography
}

Short title: Cortical development in isolated ventriculomegaly

Word count: 3.649

Number of tables: 4

Number of figures: 4

Authors: Nadine Hahner ${ }^{1}$, Bienvenido Puerto ${ }^{1}$, Miriam Perez-Cruz ${ }^{1}$, Catarina Policiano ${ }^{1,3}$, Elena Monterde ${ }^{1}$, Fatima Crispi ${ }^{1,2}$, Eduard Gratacos ${ }^{1,2}$, Elisenda Eixarch ${ }^{1,2}$

${ }^{1}$ Fetal i+D Fetal Medicine Research Center, BCNatal - Barcelona Center for Maternal-Fetal and Neonatal Medicine (Hospital Clínic and Hospital Sant Joan de Déu), Institut Clínic de Ginecologia, Obstetricia i Neonatologia, Institut d'Investigacions Biomèdiques August Pi i Sunyer, Universitat de Barcelona, Spain

${ }^{2}$ Centre for Biomedical Research on Rare Diseases (CIBER-ER), Barcelona, Spain

${ }^{3}$ Departamento de Obstetrícia e Ginecologia, Hospital de Santa Maria, Centro Hospitalar Lisboa Norte, Lisboa, Portugal.

\section{Corresponding author:}

Eduard Gratacos

BCNatal - Barcelona Center for Maternal-Fetal and Neonatal Medicine.

Sabino de Arana 1, 08028 Barcelona, Spain.

Phone: +34 93227 9333; Fax: +34 932275612

E-mail: gratacos@clinic.cat

\section{Conflict of interest statement}

No conflict of interest

This article has been accepted for publication and undergone full peer review but has not been through the copyediting, typesetting, pagination and proofreading process which may lead to differences between this version and the Version of Record. Please cite this article as doi: $10.1002 / p d .5240$ 


\section{Funding statement}

This project has been funded with support of the Erasmus + Programme of the European Union (Framework Agreement number: 2013-0040), Instituto de Salud Carlos III (PI13/01018, PI16/00861, and INT16/00168) integrados en el Plan Nacional de I+D +I y cofinanciados por el ISCIII-Subdirección General de Evaluación y el Fondo Europeo de Desarrollo Regional (FEDER) "Una manera de hacer Europa"; "la Caixa" Foundation, The Cerebra Foundation for the Brain-Injured Child, Carmarthen, Wales and Bolsa Interno Doutorando FCT - SFRH/SINTD/92997/2013. This publication reflects the views only of the author, and the Commission cannot be held responsible for any use.

\section{What's already known about this topic?}

- Fetuses with isolated non-severe ventriculomegaly are at risk of neurodevelopmental delay.

- Calcarine and parieto-occipital fissure have been demonstrated to present differential development in ventriculomegaly.

\section{What does the study add?}

- This study provides a comprehensive evaluation of cortical development in isolated non-severe ventriculomegaly including regions that have not been explored previously.

- This study demonstrates that fetuses with isolated non-severe ventriculomegaly have underdeveloped cortical maturation, also in regions that are unlikely to be affected by mechanical effect due to ventricular dilation, including Sylvian fissure, mesial area and cingulate sulcus. 


\section{ABSTRACT}

Objectives To perform a comprehensive assessment of cortical development in fetuses with isolated non-severe ventriculomegaly (INSVM) by neurosonography.

Methods We prospectively included 40 fetuses with INSVM and 40 controls. INSVM was defined as atrial width between 10.0 and $14.9 \mathrm{~mm}$ without associated malformation, infection or chromosomal abnormality. Cortical development was assessed by neurosonography at 26 and 30 weeks of gestation measuring depth of selected sulci and applying a maturation scale from 0 (no appearance) to 5 (maximally developed) of main sulci and areas.

Results INSVM showed underdeveloped calcarine and parieto-occipital sulci. In addition, significant delayed maturation pattern was also observed in regions distant to ventricular system including Insula depth (controls $30.8 \mathrm{~mm}$ (SD 1.7) vs INSVM $31.7 \mathrm{~mm}(1.8) ; p=0.04$ ), Sylvian fissure grading (>2 at 26 weeks: controls $87,5 \%$ vs INSVM 50\%, p=0.01), mesial area grading (>2 at 30 weeks: controls $95 \%$ vs INSVM 62,5\%; $p=0.03$ ), and cingulate sulcus grading ( $>2$ at 30 weeks: controls $100 \%$ vs INSVM 80,5\%; $p=0.01$ ).

Conclusions Fetuses with INSVM showed underdeveloped cortical maturation including also regions, where effect of ventricular dilatation is unlikely. These results suggest that in a proportion of fetuses with INSVM, ventricular dilation might be related with altered cortical architecture.

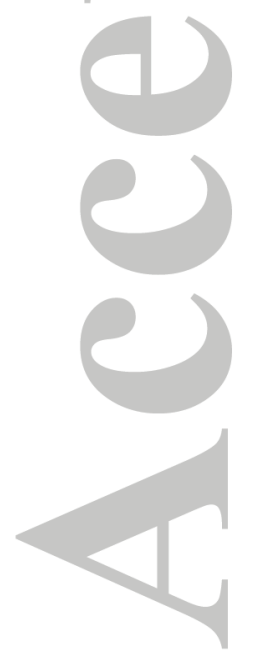

This article is protected by copyright. All rights reserved. 


\section{INTRODUCTION}

Ventriculomegaly (VM) is the most frequent brain abnormality diagnosed in fetal live occurring in about $1 \%$ of fetuses ${ }^{1}$. VM is diagnosed when atrial width is $10 \mathrm{~mm}$ or more in at least one of the lateral ventricles measured by ultrasound ${ }^{2}$. In around $50 \%$ of cases associated abnormalities, including intracranial or extracranial malformations, chromosomal anomalies or fetal infections, are found, which determines essentially their prognosis ${ }^{3}$. If no other alterations are detected, VM is considered as isolated and has in general good prognosis. Nonetheless, about $11 \%$ of these fetuses will present neurobehavioral problems $^{3-5}$, comprising wide range of possible alterations, including motor ${ }^{6,7}$, language ${ }^{8,9}$, cognitive $e^{6,7,10}$ and behavioral dysfunctions ${ }^{6-8}$. Furthermore also psychiatric disorders has been associated to ventriculomegaly, such as autism ${ }^{11,12}$, schizophrenia ${ }^{13,14}$ and attention deficit hyper activity disorder ${ }^{15}$. Nowadays the only prognostic markers to identify fetuses with higher risk for adverse outcome are ventricle width ${ }^{16}$ and progression of dilatation ${ }^{9,17}$, but to differentiate those cases that will present neurodevelopmental impairments in the group of isolated non-severe ventriculomegaly (INSVM), where most cases remains stable, more specific markers are needed.

Few studies have explored changes in cortical development in isolated mild VM, but their results are contradictory ${ }^{18-23}$. Evaluation of cortical volumes by magnetic resonance imaging (MRI) has shown increased cortical volume in fetal period ${ }^{18}$ and neonates ${ }^{24,25}$, which were persisting until two years of age ${ }^{19}$. However, in a cohort of early gestational age these differences could not be detected ${ }^{20}$. Evaluation of appearance of sulci in a non-selected population of mild fetal VM has shown significant delay of two weeks in $\mathrm{MRI}^{21}$, being the absence of specific sulci associated with poorer prognosis ${ }^{22}$. Regarding ultrasound evaluation, an inverse relationship between ventricle size and calcarine depth that correlated to cases with later progression has been described ${ }^{23}$. However, these studies did not quantify cortical maturation or only included few sulci in their evaluation missing other brain regions that could be also affected. In addition, some of them have included severe cases and non-isolated cases, two conditions that can bias their results. Thus, a complete evaluation of cortical development using quantifiable methodologies in a truly INSVM is needed. 
The objective of this work was to systematically explore cortical folding in fetuses with INSVM by means of neurosonography.

\section{METHODS}

\subsection{Subjects}

A prospective cohort study including singleton fetuses with non-severe VM attended at the Neurosonography Unit in BCNatal (Hospital Clínic and Hospital Sant Joan de Déu) in Barcelona from 2014 to 2016. Non-severe VM was defined as an atrial width between 10.0 and $14.9 \mathrm{~mm}$. Cases that progress over $14.9 \mathrm{~mm}$ or that had a previous atrial measurement $\geq 15 \mathrm{~mm}$ were excluded. Amniocentesis for evaluation of genetic anomalies and cytomegalovirus (CMV) infection was offered to all cases. Maternal blood serology for CMV was also performed. All patients with, abnormal karyotype or microarray, infections or other structural abnormalities associated with higher risk of abnormal neurodevelopment (including sulcation or migration disorders) were excluded. Control fetuses were singleton low-risk pregnancies with normally grown fetuses without structural abnormalities attended at BCNatal, and frequency matched with cases by gestational age at scan ( \pm 1 week). Controls did not undergo any additional genetic testing or examination of infection apart from routine blood testing during pregnancy. The Ethics Committee from our center (HCB/2014/0484), approved the study protocol and all patients gave written informed consent.

\subsection{Neurosonography}

Ultrasound evaluation was performed in our neurosonography unit at the Hospital Clínic Barcelona using a GE Voluson 8 Expert (GEHealthcare, London, UK) at two time-points: 26 and 30 weeks of gestation. Depending on gestational age at moment of inclusion one or two examinations were scheduled. In these two scans, we performed a multiplanar neurosonography including transabdominal and transvaginal ultrasound in cases with cephalic presentation. All examinations were done by two observers (N.H. and E.E.). Additional ultrasound images were stored for later off-line analysis including standard neurosonographic axial, sagittal and coronal planes and the plane slightly superior to the axial transventricular view ${ }^{26,27}$. Clips including axial, sagittal and coronal sweeps were stored. During ultrasound examination laterality was assessed and marked according to the 
fetal position in utero. Also, special care was taken in order to avoid as much shadowing as possible and to obtain a symmetric position of both hemispheres.

As general neurosonographic measurements, we obtained in the transthalamic axial plane biparietal diameter (BPD), head circumference $(\mathrm{HC})^{26}$, and the third ventricular width by drawing a perpendicular line between the inner borders of both lateral walls ${ }^{28}$. In the transventricular plane, atrial width was obtained at the level of the parieto-occipital sulcus (POS) by placing the calipers in the inner part of the wall perpendicular to ventricular cavity $^{26}$. The proximal lateral ventricle was assessed using coronal planes ${ }^{29}$. Progression or regression was defined as a change of lateral ventricular width of $\geq 2 \mathrm{~mm}$ between ultrasound examination at diagnosis and last ultrasound of clinical follow up during pregnancy.

Cerebellum and posterior fossa were assessed in the axial transcerebellar plane. The cerebellar diameter was measured drawing a perpendicular line between the most lateral points of each hemisphere and the cisterna magna depth was measured from vermis to the inner border of the skull ${ }^{26}$. The sagittal and coronal views were obtained both by transvaginal approach in cases of cephalic position and transabdominally in fetuses with breech position. In the midsagittal plane length of the corpus callosum was measured by drawing a line from the most anterior part of the genu to the most posterior of the splenium $^{30}$. In the same plane, cranio-caudal diameter of the vermis was measured ${ }^{31}$. To obtain complete ventricular assessment, cranio-caudal diameter of anterior horns were measured in the transcaudate coronal plane from inner to inner border and perpendicular to the walls of both anterior horns ${ }^{32,33}$.

\subsection{Sulcal depth}

Sulci measurement was performed offline using Alma Workstation 4.2.0.25 (2005 - 2014 Alma IT Systems, S.L. All rights reserved, Barcelona Spain). To provide rigorous perpendicular measurements to the midline, a straight line projecting the interhemispheric fissure was traced in every plane going from frontal bone to occipital bone in axial views and from cranial to caudal bone in coronal views. Sulci of both hemispheres were measured in $\mathrm{mm}$ and the values were corrected by the BPD and multiplied by 100 in order to normalize by head size $e^{34}$. Approach of sulci measurements applying the methodology of Egaña et $\mathrm{al}^{34}$ and Alonso et al. ${ }^{35}$ is depicted in Figure 1. 
Starting with the axial views, Insula depth was measured in the transthalamic plane drawing a perpendicular line from midline directly behind the Cavum septi pellucidi to the external border of the cortex ${ }^{34}$. To measure the Sylvian fissure we continued the line of the Insula depth with the same degree starting the measurement at the external border of the cortex and terminating at the internal border of the cranium (Figure 1a) ${ }^{34}$. POS depth was measured in a slightly superior plane above the transventricular plane starting a perpendicular line from midline and ending at the apex of the fissure without including de cortex (Figure $1 b)^{34}$. To measure the cingulate sulcus depth the transthalamic plane in the coronal view was used and a perpendicular line was drawn from midline until the apex of the cingulate sulcus without including the cortex (Figure 1c). Calcarine sulcus was also measured in the coronal view using the transcerebellar plane and tracing a perpendicular line from midline to the apex of the sulcus not including the cortex. (Figure 1d).

\subsection{Cortical grading}

We classified the maturation of main sulci and areas using the scoring method as described by Pistorius et al. ${ }^{27}$ and shown in Figure 1 . Three grading schemes were used to grade specific areas (frontal, parietal, temporal, and mesial cortical area), sulci (parieto- occipital, superior temporal, central, calcarine and cingulate sulcus) and the Sylvian fissure. The score ranged their maturation into 5 grades, where 0 was no development and 5 corresponded to the highest grade of maturation. Ideally both hemispheres were evaluated, which was feasible for most sulci and mesial area, but not possible for areas and sulci of the proximal cortical surface.

In the axial transthalamic plane we obtained the grading of the Sylvian fissure, superior temporal sulcus and temporal area (Figure 1e). In the axial plane parallel and slightly above the transventricular plane we evaluated the grading of the parieto-occipital and central sulcus, frontal and parietal area (Figure $1 \mathrm{f})^{27}$.

In the coronal transthalamic plane we assessed cingulate sulcus and mesial area (Figure 1g). The Calcarine sulcus was obtained in a coronal transcerebellar plane (Figure $1 \mathrm{~h})^{27}$.

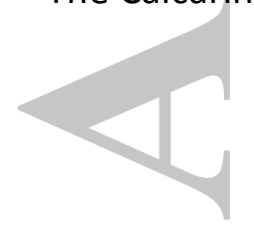




\subsection{Statistical analysis}

Data analyses were performed using SPSS version 21 for Mac (SPSS Inc. Chicago, IL, USA). Student $\mathrm{t}$ test for independent samples and Pearson's Chi-square tests were used to compare quantitative and qualitative data, between INSVM and controls. For quantitative variables, multivariate analyses of covariance were conducted, adjusting by gender and gestational age at ultrasound. For ordinal variables, ordinal regression was performed adjusting by gender and gestational age at ultrasound.

To determine intra- and interobserver variability of depth measurements and grading scores, 20 patients (10 of each time point) were evaluated by two blinded observers (N.H. and C.P.). For the intraobserver variability, one operator (N.H.) has evaluated the same patient after at least one month without being aware of the initial values and Intraclass correlation coefficient (ICC) was calculated using two-way mixed model with absolute agreement. For the interobserver variability, two operators (N.H. and C.P.) have measured the same patient being blinded for the measurements and ICC was calculated using two-way random effect model with absolute agreement. Images of examinations were not specifically selected for re-evaluation of each variable.

\section{RESULTS}

\subsection{Subjects}

Forty-two VM cases with no associated brain malformations were included. One VM case was excluded due to a positive PCR of cytomegalovirus in amniotic fluid and another due to a 16p11.2 duplication detected in the micro-array and associated with a high risk of neurodevelopmental disorder, leaving a final sample size of 40 cases of INSVM and 40 controls. Normal karyotype $(n=23)$ or micro-array $(n=14)$ was found in all patients that underwent amniocentesis ( $n=37)$. In three cases, patients refused amniocentesis, but did not present any sign of genetic disorders after birth and their routine first trimester screening indicated low risk for Down and Edwards syndrome. Infection was excluded by negative $\mathrm{PCR}$ of $\mathrm{CMV}$ in amniotic liquid in 34 cases and in the rest by maternal serologic screening. No additional findings were detected postnatally in any case. Demographic characteristics of the study groups are presented in Table 1. INSVM group had significantly more male fetuses than the control group, but no difference was found within ventricular 
size between males and females in case group (INSVM males: $10.6 \mathrm{~mm} \pm 1.5 \mathrm{~mm}$ vs INSVM females: $11.2 \pm 0.6 \mathrm{~mm} ; p=0.39)$.

\subsection{Neurosonography}

All cases and controls underwent at least one neurosonography. 35 INSVM cases had ultrasound scan at two-time points, and accordingly 35 controls had the same two examinations. As 5 VM cases were referred at later gestational age and only the 30 weeks scan was performed, 5 controls were additionally included to complete the sample size. Apart from the cortical folding, main cerebral structures were evaluated at 26 and 30 weeks scan. The measurements of both groups are depicted in Table 2. As expected, left and right lateral ventricle of cases showed significant greater width than controls with a 2-fold increase in mean values in both hemispheres. Additionally, cranio-caudal diameter of anterior horns showed increased size in INSVM $(p<0.01)$ and also the third ventricle was significantly wider $(p<0.01)$. In both examinations, no significant differences were found in BPD, cerebellar diameter, posterior fossa width, corpus callosum length and cranio-caudal diameter of vermis.

\subsection{Sulcal depth}

In general, INSVM showed decreased sulcal depth compared to controls. These differences were subtle at 26 weeks of gestation. At this moment the few significant changes were present in calcarine and POS and the right Insula in the bilateral subgroup (Table 3). However, at 30 weeks scan more evident alterations were observed (Table 4). Differences in parieto-occipital and calcarine sulci measurement, both next to the lateral ventricles, were stronger than in previous examination and also present in the contralateral hemisphere. In addition, significant changes in right Sylvian fissure and in the left Insula could be identified. It should be noted that, at 30 weeks, changes were more evident in the right hemisphere compared with left, including both bilateral and unilateral cases.

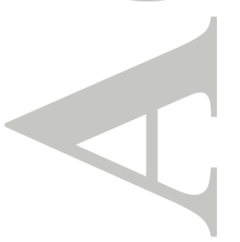




\subsection{Cortical grading}

Overall fetuses with INSVM showed underdeveloped cortical maturation compared to controls, being these changes more pronounced at 30 weeks. At 26 weeks, INSVM cases showed significant differences with more mature pattern in left frontal area and in Sylvian fissure, calcarine sulcus and POS presenting underdeveloped maturation compared to the control group (Figure 2). When analyzing subgroups separately, bilateral cases showed changes in right mesial area and unilateral-right cases in right superior temporal sulcus. Alterations in calcarine sulcus maturation were observed in all subgroups, being in unilateral cases only present in the ipsilateral side. Interestingly, at 30 weeks changes involved other regions, including regions distant to posterior horn of lateral ventricles. Left mesial area and cingulate sulcus were significantly less developed when compared to controls (Figure3). To note, the advanced maturation of frontal area observed at 26 weeks was not identified at 30 weeks scan. On the contrary, cases had lower grading scores than controls, but without reaching statistical significance. When analyzing subgroups, changes in cingulate sulcus and left mesial area were only observed in bilateral cases. Furthermore, right mesial area appeared to be less developed in the unilateral-right INSVM group.

\subsection{Intra- and Interobserver agreement}

Complete evaluation of cortical grading and sulci depth could be performed in more than $90 \%$ of subjects.

Overall, cortical development assessment showed a good intra- and interobserver reproducibility. Regarding depth measurements, intra- and interobserver variability showed an ICC of 0.926 and 0.892 for Insula, 0.897 and 0.878 for Sylvian fissure, 0.836 and 0.773 for POS, 0.971 and 0.917 for cingulate sulcus, and 0.685 and 0.782 for calcarine sulcus.

Regarding grading scores of areas and sulci, intra- and interobserver variability were 0.925 and 0.800 for fontal area, 0.828 and 0.889 for parietal area, 0.846 and 0.813 for temporal area, 0.896 and 0.844 for mesial area, 0.933 and 0.801 for Sylvian fissure, 0.894 and 0.906 for POS, 0.907 and 0.897 for central sulcus, 0,890 and 0.762 for superior temporal sulcus, 0.955 and 0.765 for cingulate sulcus and 0.894 and 0.767 for calcarine sulcus grading. 


\section{DISCUSSION}

To the best of our knowledge this is the first study, which conducted a comprehensive and detailed evaluation of cortical development using ultrasound in fetuses with truly INSVM. This study demonstrated changes in cortical development, not only in regions next to the ventricles, but also in regions that are not directly affected by a potential mechanical effect of ventricular dilatation, such as Sylvian fissure, mesial area and cingulate sulcus. These findings suggested that INSVM could be sensitive marker of altered neurodevelopment expressed by underdeveloped cortical maturation.

As far as we know this is the first study describing changes in cortical development in Sylvian fissure, mesial area and cingulate sulcus using ultrasound. We hypothesized that larger Insula and less developed Sylvian fissure found in our population could explain the altered neurodevelopment observed in a proportion of children with ventriculomegaly. Functionally, Sylvian fissure and Insula are described to be involved in speech production $^{36,37}$, emotion and social awareness ${ }^{38,39}$, thus changes observed in this region could explain neurodevelopmental disfunctions observed in this population. Additionally, we have observed significant underdevelopment in mesial area and cingulate sulcus maturation. Mesial area and cingulate cortex are described to interact in a wide range of functions including emotional ${ }^{40}, \operatorname{cognitive}^{41}$, motor $^{42}$ and behavioral ${ }^{43}$ processes. Also in psychiatric disorders such as schizophrenia, attention deficit hyperactivity disorder and autism, alterations in this area could be found ${ }^{44-47}$. Indeed, identification of the cingulate fissure using MRI at the moment of diagnosis has been demonstrated to be related with good prognosis ${ }^{22}$. However, alterations in mesial region might not be related just with the presence of cingulate sulci. In our cohort, we found that some INSVM cases showed wider interhemispheric space above cingulate sulci (Figure 4), which correspond to the anterior part of superior frontal gyrus. This region is highly connected with cingulate cortex and involved in cognitive control and in the default mode network, which is essential for normal cognition and self-referential processing ${ }^{48}$.

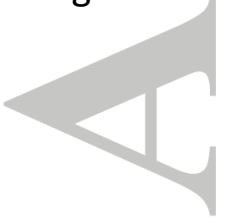


Our findings in parieto-occipital and calcarine sulcus, both close to the posterior horns of the lateral ventricles, are in line with previous studies showing less profound and developed sulci $23,49,50$, although none of them explored the effect of ventricular dilation in contralateral hemisphere. On this regard, we demonstrated that calcarine sulcus was less deep in the hemisphere contralateral to ventricular dilation in those cases with unilateral dilation. Changes in parieto-occipital and calcarine sulci have been previously related with ventricular enlargement, though the mechanism behind is still controversial ${ }^{23}$. Decreased sulci depth could be related with a mechanical deformation due to ventricular dilation or may indicate an underdeveloped maturation due to altered neuronal proliferation and migration. Due to the existence of changes in contralateral hemisphere and other cortical regions far from ventricular system, we hypothesized that these changes are not explained only by mechanical deformation. During normal brain development ventricular and germinal matrix volumes are closely related, they increases in the first half of the pregnancy and decreases after 23 weeks of pregnancy ${ }^{49,51}$. It could be suggested that, in some cases of INSVM, changes in the migration process may be the cause of both disruption of normal ventricular volume reduction and altered cortical maturation. This could also explain the fact that INSVM have shown increased grey matter volumes during fetal period ${ }^{18}$ and up to 2 -years of ages ${ }^{19}$, which could be related with increased proliferation and changes in cortical cytoarchitecture. Interestingly, when analyzing subgroups of INSVM more alterations were found in bilateral cases, although most studies describe a similar risk of neurodevelopmental delay in bilateral and unilateral INSVM ${ }^{4,9}$. Comparison between regressive and stable INSVM cases showed similar cortical development parameters, which emphasize the notion that regressive cases might be also marker for altered brain development. Due to small sample sizes of these subgroups the results are limited and more likely a tendency is reflected.

We acknowledge that our study has strengths and limitations that deserve some discussion. Among the strengths of this study, both groups were included prospectively and INSVM was a rigorous selected population with normal karyotype or microarray and without infection or structural malformations. In addition, all cases and controls underwent serial neurosonography to exclude any additional abnormality of the central nervous system. These results in a group of truly INSVM, preventing the inclusion of some conditions that 
could potentially altered brain development and bias our results. Instead of focusing on only one or two brain regions, the evaluation of multiple areas provides a more comprehensive picture of changes in cortical development. In addition, the use of both depth measurement and grading is an advantage of this study. Measurement of sulci depth gives quantitative and objective values that can be easily performed, but do not inform about maturation status. On the contrary, cortical grading gives important information about brain areas and sulci maturation, but it consists only in five grades and subtle differences might not been captured, especially in advanced maturation status. To provide high image quality and precise assessment of cortical development parameters, ultrasound was performed by two trained and experienced neurosonographers following a strict protocol, which is crucial in order to obtain correct planes and reliable measurements. As a limitation, we admit that our measurements and grading were done manually since there is no automatic software to perform this type of analysis. To overcome this issue, all measurements have been done by the same examiner (N.H.), following a strict protocol in order to identify exact planes to assess the parameters. Indeed, all measurements showed acceptable intra- and interobserver agreement. Secondly, although ultrasound enables detailed evaluation of fetal brain structures, some regions could be hidden by shadowing from fetal skull. We acknowledge that additional information could be provided by MRI, especially after 32 weeks of gestation, which do not have this limitation and allows detailed evaluation of cortical development in both hemispheres. However, our aim was to use a methodology that is more widely available to obstetricians and that can be used during clinical practice. Furthermore, MRI is not included in the study protocol of INSVM in all centers. Thirdly, it should be stressed that differences in folding pattern identified in our cases were subtle changes, so we cannot provide any clinical cut off value for implementation in clinical practice and, more importantly, it cannot be considered as sulcation disorder. Finally, to confirm weather changes in cortical development obtained prenatally correlates with poorer neurobehavior observed in a proportion of INSVM cases, further data about neurodevelopment is needed. 
From clinical point of view, results of this study provide new evidence about the existence of prenatal changes in cortical development in fetuses with INSVM that could be of help to identify those cases at higher risk of altered neurodevelopment. Our study considered a sheer cohort of INSVM fetuses excluding all those cases with additional malformations, infection or chromosomal aberration. Findings in this subgroup are of great relevance for clinical practice as it is a frequent finding of the central nervous system and the current prognostic markers ventricular width and progression are insufficient to select those cases that will develop neurodevelopmental problems. Evaluation of sulci depth and grading is a feasible method to apply in clinical settings giving global overview of the maturation status of the entire cortex, which is important to also detect regional alterations. Longitudinal evaluation of cortical development in INSVM could help in the understanding of these changes and to evaluate whether these differences persist later in pregnancy.

\section{CONCLUSION}

Fetuses with INSVM showed delayed cortical folding that could be reliably assessed by neurosonography, including regions as Sylvian fissure, mesial area and cingulate sulcus that are unlikely to be affected by mechanical effect of ventricular dilatation. Our results support the hypothesis that in a proportion of fetuses with INSVM, ventricular dilation could be related with altered proliferation and migration processes leading to changes in cortical architecture. We propose assessment of cortical development using ultrasound as possible biomarker that could select those fetuses with high risk of poorer neurodevelopmental outcome. Further studies are needed to evaluate the potential utility of these fetal ultrasound parameters to predict neurodevelopmental outcome. 


\section{ACKNOWLEDGEMENTS}

The authors gratefully acknowledge the parents who participated in this study.

\section{FUNDIGN SOURCES:}

This project has been funded with support of the Erasmus + Programme of the European Union (Framework Agreement number: 2013-0040). This publication reflects the views only of the author, and the Commission cannot be held responsible for any use, which may be made of the information contained therein. This study was also supported by Instituto de Salud Carlos III (PI13/01018, PI16/00861, and INT16/00168) integrados en el Plan Nacional de I+D+I y cofinanciados por el ISCIII-Subdirección General de Evaluación y el Fondo Europeo de Desarrollo Regional (FEDER) "Una manera de hacer Europa"; "la Caixa” Foundation, and The Cerebra Foundation for the Brain-Injured Child, Carmarthen, Wales. C.P. was supported by Bolsa Interno Doutorando FCT - SFRH/SINTD/92997/2013.

\section{REFERENCES}

1. Salomon LJ, Bernard JP, Ville Y. Reference ranges for fetal ventricular width: A nonnormal approach. Ultrasound Obstet Gynecol 2007; 30: 61-66.

2. Cardoza JD, Goldstein RB, Filly RA. Exclusion of fetal ventriculomegaly with a single measurement: the width of the lateral ventricular atrium. Radiology 1988; 169: 7114.

3. Leung WC, Pandya P, Seaward G, et al. Mild ventriculomegaly in the fetus, natural history, associated findings and outcome of isolated mild ventriculomegaly: A literature review. Prenat Diagn 2001; 21: 697-700.

4. Melchiorre K, Bhide A, Gika AD, et al. Counseling in isolated mild fetal ventriculomegaly. Ultrasound Obstet Gynecol 2009; 34: 212-224.

5. Moeschler JB, Shevell M. Clinical genetic evaluation of the child with mental retardation or developmental delays. Pediatrics 2006; 117: 2304-2316.

6. Gómez-Arriaga P, Herraiz I, Puente JM, et al. Mid-term neurodevelopmental outcome in isolated mild ventriculomegaly diagnosed in fetal life. Fetal Diagn Ther 2012; 31: $12-18$.

7. Leitner $\mathrm{Y}$, Stolar $\mathrm{O}$, Rotstein $\mathrm{M}$, et al. The neurocognitive outcome of mild isolated 
fetal ventriculomegaly verified by prenatal magnetic resonance imaging. Am J Obstet Gynecol 2009; 201: 215.e1-215.e6.

8. Falip C, Blanc N, Maes E, et al. Postnatal clinical and imaging follow-up of infants with prenatal isolated mild ventriculomegaly: A series of 101 cases. Pediatr Radiol 2007; 37: 981-989.

9. Ouahba J, Luton D, Vuillard E, et al. Prenatal isolated mild ventriculomegaly: Outcome in 167 cases. BJOG An Int J Obstet Gynaecol 2006; 113: 1072-1079.

10. Bloom SL, Bloom DD, Dellanebbia C, et al. The developmental outcome of children with antenatal mild isolated ventriculomegaly. Obstetrics and Gynecology 1997; 90: 93-97.

11. Palmen SMC, Hulshoff Pol HE, Kemner C, et al. Increased gray-matter volume in medication-naive high-functioning children with autism spectrum disorder. Psychol Med 2005; 35: 561-570.

12. Piven J, Bailey J, Havercamp S, et al. An MRI study of brain size in autism. Am J Psychiatry 1995; 152: 1145-1149.

13. Wright IC, Rabe-Hesketh S, Woodruff PWR, et al. Meta-analysis of regional brain volumes in schizophrenia. Am J Psychiatry 2000; 157: 16-25.

14. Gilmore JH, Van Tol J, Kliewer MA, et al. Mild ventriculomegaly detected in utero with ultrasound: Clinical associations and implications for schizophrenia. Schizophr Res 1998; 33: 133-140.

15. Lyoo IK, Noam GG, Lee CK, et al. The Corpus Callosum and Lateral Ventricles in Children with Attention-Deficit Hyperactivity Disorder : A Brain Magnetic Resonance Imaging Study. Biol Psychiatry 1996; 40: 1060-1063.

16. Vergani P, Locatelli A, Strobelt N, et al. Clinical outcome of mild fetal ventriculomegaly. Am J Obstet Gynecol 1998; 178: 218-222.

17. Gaglioti P, Danelon D, Bontempo S, et al. Fetal cerebral ventriculomegaly: Outcome in 176 cases. Ultrasound Obstet Gynecol 2005; 25: 372-377.

18. Kyriakopoulou V, Vatansever D, Elkommos S, et al. Cortical Overgrowth in Fetuses With Isolated Ventriculomegaly. Cereb Cortex 2014; 24: 2141-2150.

19. Lyall $A E$, Woolson $\mathrm{S}$, Wolfe $\mathrm{HM}$, et al. Prenatal isolated mild ventriculomegaly is associated with persistent ventricle enlargement at ages 1 and 2. Early Hum Dev 2012; 88: 691-8. 
20. Scott JA, Habas PA, Rajagopalan V, et al. Volumetric and surface-based 3D MRI analyses of fetal isolated mild ventriculomegaly: brain morphometry in ventriculomegaly. Brain Struct Funct 2013; 218: 645-55.

21. Levine D, Barnes PD. Cortical Maturation in Normal and Abnormal Fetuses as Assessed with prenatal MR Imaging. Radiology 1999; 210: 751-758.

22. Li Y. Ultrasound and MRI of fetuses with Ventriculomegaly: can Cortical Development be used to predict Postnatal outcome. 2012; 196: 1457-1467.

23. Miguelote RF, Vides B, Santos RF, et al. Cortical maturation in fetuses referred for 'isolated' mild ventriculomegaly: a longitudinal ultrasound assessment. Prenat Diagn 2012; 32: 1273-81.

24. Lockwood Estrin G, Kyriakopoulou V, Makropoulos A, et al. Altered white matter and cortical structure in neonates with antenatally diagnosed isolated ventriculomegaly. Neurolmage Clin 2016; 11: 139-148.

25. Gilmore JH, Smith LC, Wolfe HM, et al. Prenatal Mild Ventriculomeglay Predicts Abnormal Development of Neonatal Brain. 2009; 64: 1069-1076.

26. Sonographic examination of the fetal central nervous system: guidelines for performing the 'basic examination' and the 'fetal neurosonogram'. Ultrasound Obstet Gynecol 2007; 29: 109-16.

27. Pistorius LR, Stoutenbeek P, Groenendaal F, et al. Grade and symmetry of normal fetal cortical development: a longitudinal two- and three-dimensional ultrasound study. Ultrasound Obstet Gynecol 2010; 36: 700-8.

28. Sari A, Ahmetoglu A, Dinc H, et al. Fetal biometry: size and configuration of the third ventricle. Acta Radiol 2005; 46: 631-635.

29. Katorza E, Duvdevani N, Jacobson JM, et al. Coronal approach for measuring both fetal lateral ventricles: Is there an advantage over the axial view? Prenat Diagn 2014; 34: $279-284$.

30. Achiron R, Achiron A. Development of the human fetal corpus callosum: A highresolution, cross-sectional sonographic study. Ultrasound Obstet Gynecol 2001; 18: $343-347$.

31. Benn PA, Gainey A, Ingardia CJ, et al. The fetal cerebellar vermis: Normal development as shown by transvaginal ultrasound. Prenat Diagn 2001; 21: 687-692.

32. Brouwer MJ, de Vries L, Groenendaal F, et al. New Reference Values for the Neonatal 
Cerebral Ventricles. Radiology 2012; 262: 224-233.

33. Davies MW, Swaminathan M, Chuang SL, et al. Reference ranges for the linear dimensions of the intracranial ventricles in preterm neonates. Arch Dis Child Fetal Neonatal Ed 2000; 82: F218-23.

34. Egaña-Ugrinovic G, Sanz-Cortes M, Figueras F, et al. Differences in cortical development assessed by fetal MRI in late-onset intrauterine growth restriction. Am J Obstet Gynecol 2013; 209: 126.e1-8.

35. Alonso I, Borenstein M, Grant G, et al. Depth of brain fissures in normal fetuses by prenatal ultrasound between 19 and 30 weeks of gestation. Ultrasound Obstet Gynecol 2010; 36: 693-9.

36. Craig ADB. Significance of the insula for the evolution of human awareness of feelings from the body. Ann N Y Acad Sci 2011; 1225: 72-82.

37. Ogar J, Willock S, Baldo J, et al. Clinical and anatomical correlates of apraxia of speech. Brain Lang 2006; 97: 343-350.

38. Picard F. State of belief, subjective certainty and bliss as a product of cortical dysfunction. Cortex 2013; 49: 2494-2500.

39. Gasquoine PG. Contributions of the insula to cognition and emotion. Neuropsychology Review 2014; 24: 77-87.

40. Etkin A, Egner T, Kalisch R. Emotional processing in anterior cingulate and medial prefrontal. Trends Cogn Sci 2011; 15: 85-93.

41. Leech R, Sharp DJ. The role of the posterior cingulate cortex in cognition and disease. Brain 2014; 137: 12-32.

42. Picard N, Strick PL. Motor areas of the median wall: a review of their location and functional activation. Cereb Cortex 1996; 6: 342-353.

43. Hadland KA, Rushworth MFS, Gaffan D, et al. The effect of cingulate lesions on social behaviour and emotion. Neuropsychologia 2003; 41: 919-931.

44. Adam R, David AS. Patterns of anterior cingulate activation in schizophrenia: A selective review. Neuropsychiatr Dis Treat 2007; 3: 87-101.

45. Nakao T, Radua J, Rubia K, et al. Gray matter volume abnormalities in ADHD: Voxelbased meta-analysis exploring the effects of age and stimulant medication. Am J Psychiatry 2011; 168: 1154-1163.

46. Zhu CZ, Zang YF, Cao QJ, et al. Fisher discriminative analysis of resting-state brain 
function for attention-deficit/hyperactivity disorder. Neuroimage 2008; 40: 110-120.

47. Chiu PH, Kayali MA, Kishida KT, et al. Self Responses along Cingulate Cortex Reveal Quantitative Neural Phenotype for High-Functioning Autism. Neuron 2008; 57: 463473.

48. Li W, Qin W, Liu H, et al. Subregions of the human superior frontal gyrus and their connections. Neuroimage 2013; 78: 46-58.

49. Fukunishi K, Sawada K, Kashima M, et al. Correlation between formation of the calcarine sulcus and morphological maturation of the lateral ventricle in cynomolgus monkey fetuses. Acta Neurobiol Exp (Wars) 2011; 71: 381-386.

50. Li H, Liu G, Lin F, et al. Formation of the calcarine sulcus: a potential marker to predict the progression in utero of isolated mild fetal ventriculomegaly. Medicine (Baltimore) 2017; 96: e7506.

51. Kinoshita $\mathrm{Y}$, Okudera $\mathrm{T}$, Tsuru $\mathrm{E}$, et al. Volumetric analysis of the germinal matrix and lateral ventricles performed using MR images of postmortem fetuses. Am J Neuroradiol 2001; 22: 382-388.

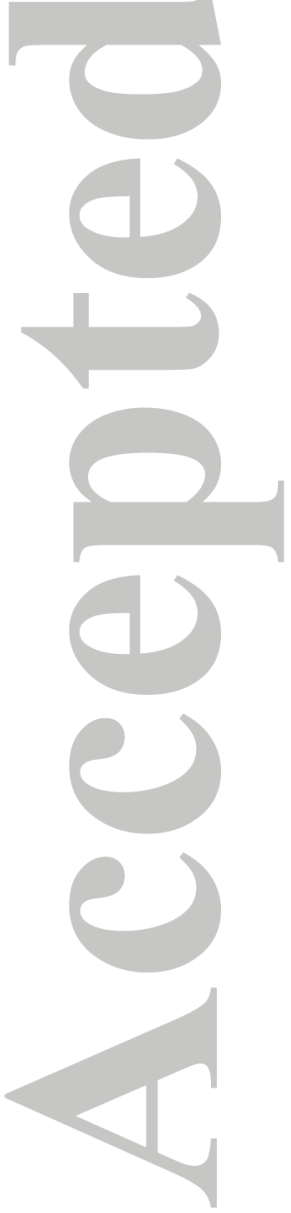




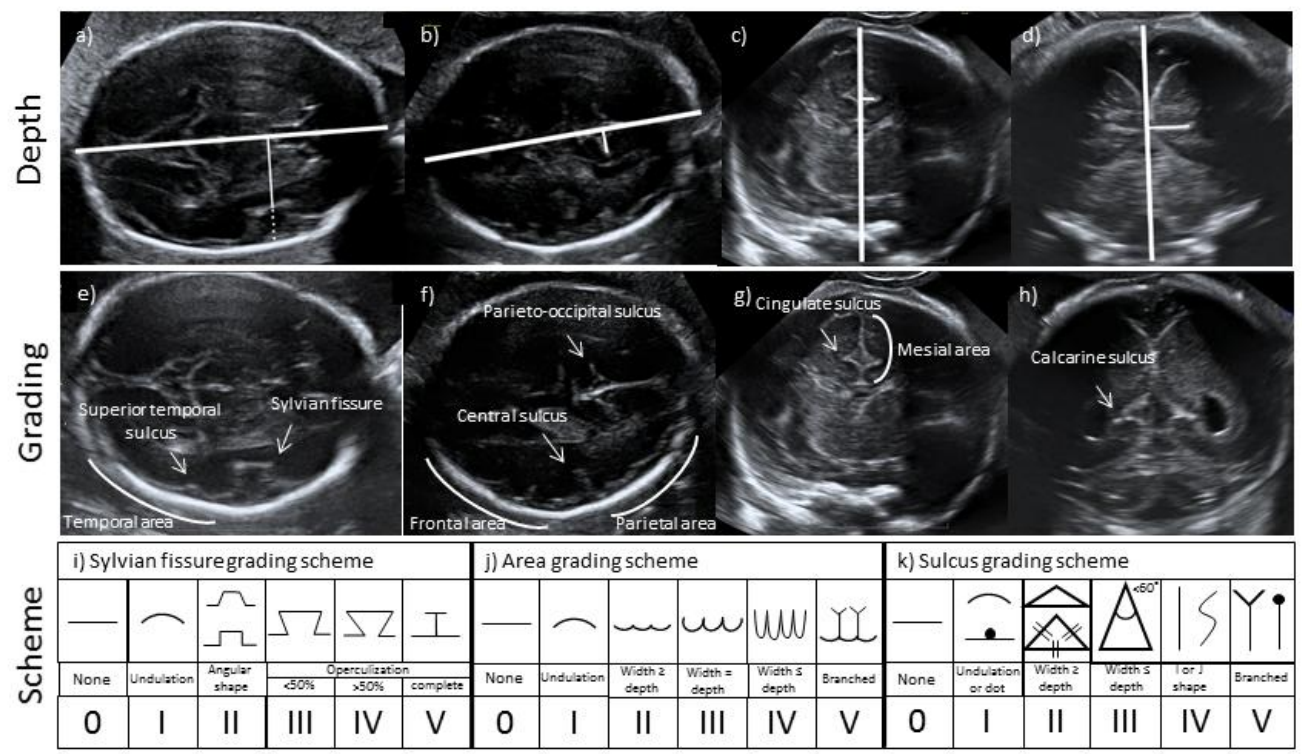

\section{Figure 1. Assessment of sulcal depth and cortical grading}

Sulcal depth: The bold white interhemispheric line is used as reference for all measurements and the thin white line shows unilateral example of measurement of sulcus of interest. a) demonstrates measurement of Insula and Sylvian fissure (dotted line), b) parieto-occipital, c) cingulate and d) calcarine sulcus.

Cortical grading: Curved lines indicate the areas and arrows point out the sulci of interest. In the transthalamic plane (e) we evaluated temporal area, Sylvian fissure and superior temporal sulcus and in the plane superior to the transventricular plane (f) frontal and parietal area and parieto-occipital sulcus. The mesial area and cingulate sulcus are presented in g) and h) shows calcarine sulcus.

Scheme: Grading score by Pistorius et al. ${ }^{24}$ 

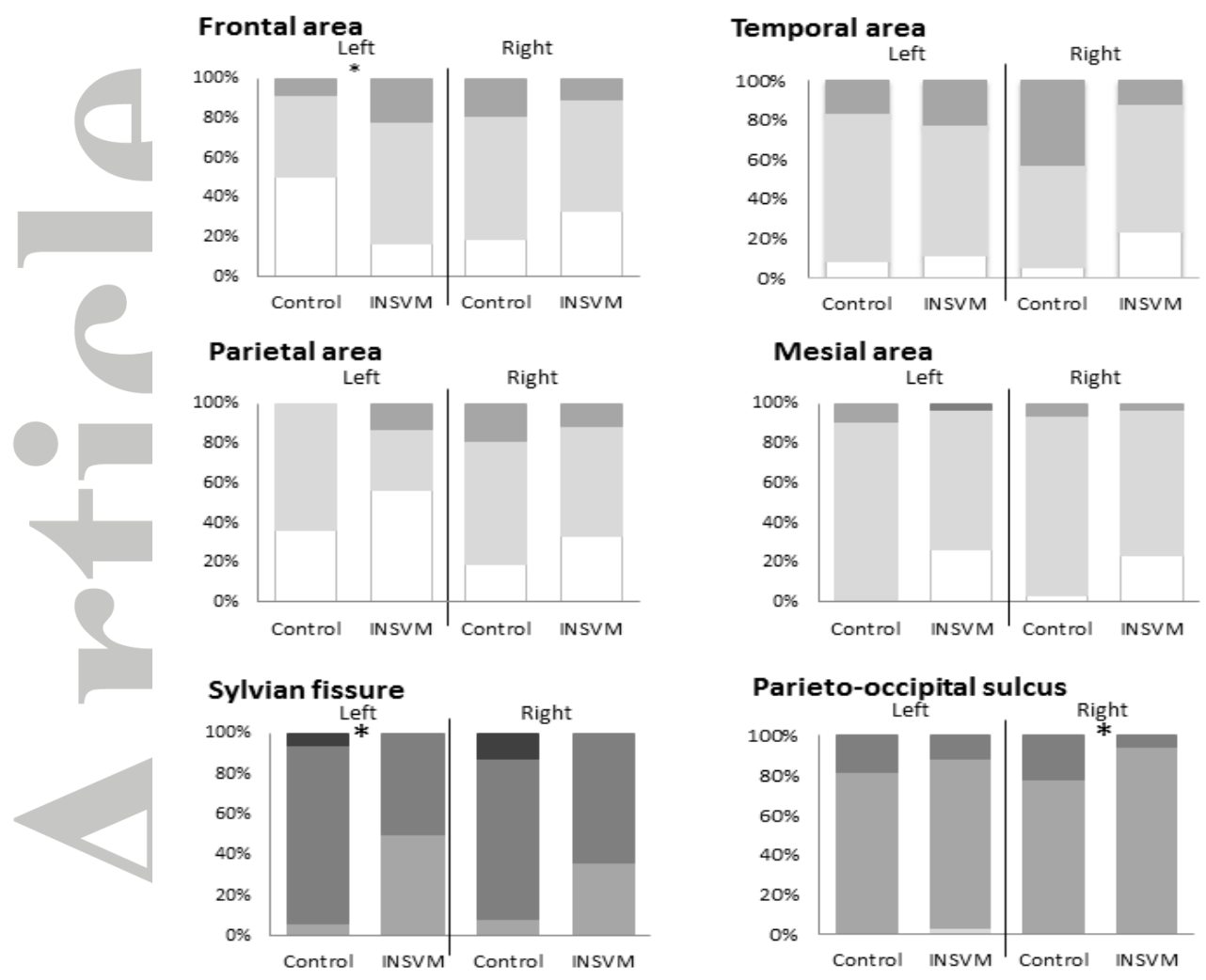

- Grading 5
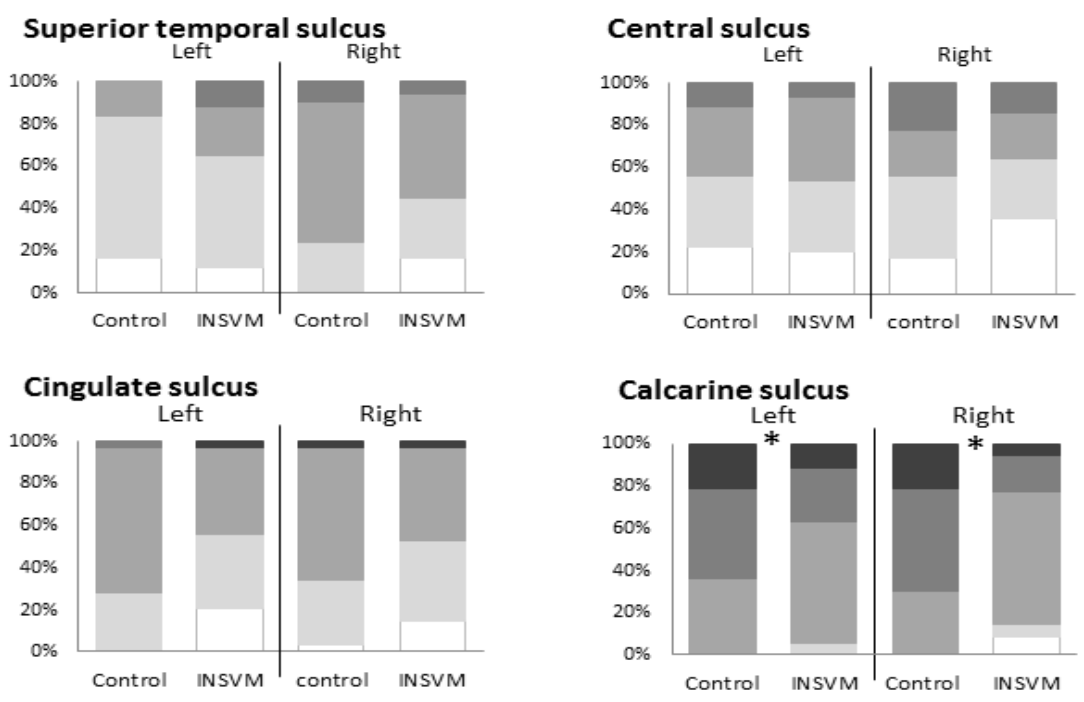

Figure 2. Cortical grading of main areas and sulci at $\mathbf{2 6}$ weeks of gestation

Distribution of cortical grading scores of main cortical areas and sulci of left and right hemisphere in INSVM and Controls at 26 weeks of gestation.

$* p \leq 0.05$

INSVM, Isolated non-severe ventriculomegaly 

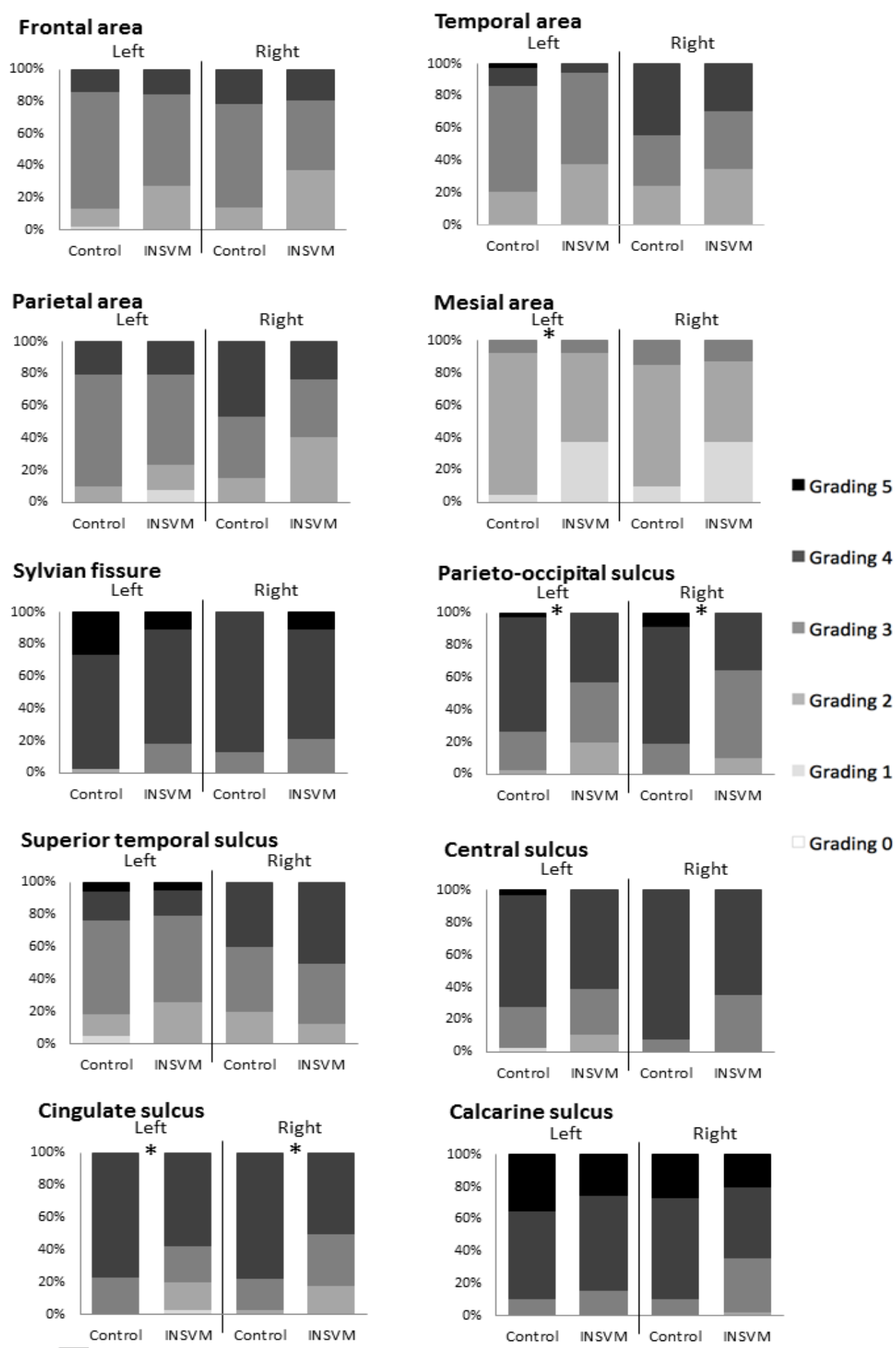

Figure 3. Cortical grading of main areas and sulci at $\mathbf{3 0}$ weeks of gestation

Distribution of cortical grading scores of main cortical areas and sulci of left and right hemisphere in INSVM and controls at 30 weeks of gestation.

$* p \leq 0.05$

INSVM, Isolated non-severe ventriculomegaly 


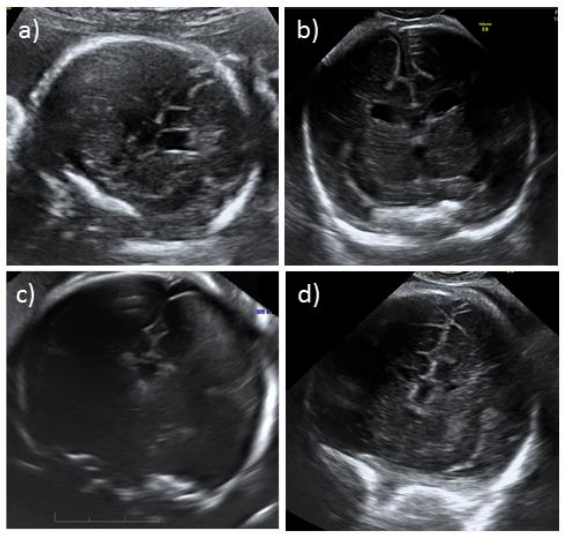

Figure 4. Mesial area and cingulate sulcus development in INSVM

Mesial area and cingulate fissure development in a coronal transthalamic view using transvaginal approach. In a) INSVM case at 27.6 weeks of gestation and b) the same case at 31.6 weeks. The images clearly show altered pattern of mesial area and cingulate sulcus maturation with increased interhemispheric space and delayed sulci development compared with a control at c) 27.4 weeks and b) 30.4 weeks gestation. 
Table 1. Demographic characteristics of the study groups

\begin{tabular}{|c|c|c|c|}
\hline Characteristic & $\begin{array}{c}\text { Control } \\
(n=40)\end{array}$ & $\begin{array}{l}\text { INSVM } \\
(n=40)\end{array}$ & $p$-Value \\
\hline Maternal age (years) & $33.8 \pm 4.2$ & $32.1 \pm 5.3$ & 0.12 \\
\hline Birth weight (g) & $3388 \pm 487$ & $3453 \pm 548$ & 0.60 \\
\hline Gestational age at birth (weeks) & $39.7 \pm 1.0$ & $39.7 \pm 1.5$ & 0.92 \\
\hline Gestational age at 26 -weeks scan (weeks) & $26.4 \pm 1.14$ & $26.4 \pm 2.9$ & 0.81 \\
\hline Gestational age at 30 -weeks scan (weeks) & $30.4 \pm 0.9$ & $30.3 \pm 3.6$ & 0.89 \\
\hline Fetus gender & & & 0.04 \\
\hline Male & $62.5 \%(25)$ & $87.5 \%(35)$ & \\
\hline Female & $37.5 \%(15)$ & $12.5 \%(5)$ & \\
\hline Distribution of subgroups & & & \\
\hline Bilateral & - & $35 \%(14)$ & \\
\hline Unilateral Left & - & $32.5 \%(13)$ & \\
\hline Unilateral Right & - & $32.5 \%(13)$ & \\
\hline Evolution of lateral ventricular width* & & & \\
\hline Regressive & - & $22.5 \%(9)$ & \\
\hline Stable & - & $77.5(31)$ & \\
\hline Progressive & - & $0 \%(0)$ & \\
\hline
\end{tabular}

Results are expressed as mean \pm SD or percentage and number of subjects as appropriate. Only gestational ages at examination time points are revealed as median \pm interquartile range. $P$-values considered as significant $(\leq 0.05)$ are marked in bold.

*Evolution of lateral ventricular width until term of pregnancy 
Table 2. Measurements of cerebral brain structures in study groups

Control

INSVM

$(n=40)$

$(n=40)$

Lateral ventricle width*

Left

Right

Anterior horns CC-diameter*

Left

Right

Third ventricle width*

BPD

$26 \mathrm{~W}$

$30 \mathrm{~W}$

Cerebellar diameter

$26 \mathrm{~W}$

$30 \mathrm{~W}$

Posterior fossa

$26 \mathrm{~W}$

Corpus Callosum length

$26 \mathrm{~W}$

$33.4 \pm 2.2$

$38.4 \pm 2.4$

$15.7 \pm 1.0$

$18.5 \pm 3.2$

$1.8 \pm 0.6$

$1.0 \pm 0.3$

$66.9 \pm 3.0$

$77.8 \pm 3.1$

$30.9 \pm 1.5$

$37.8 \pm 2.2$

$5.8 \pm 1.6$

$5.9 \pm 1.8$

$30 \mathrm{~W}$

Vermis CC-diameter

$26 \mathrm{~W}$
$30 \mathrm{~W}$

$3.3 \pm 1.4$

$<0.01$

$3.3 \pm 1.4$

$<0.01$

$1.5 \pm 0.5$

$<0.01$

$68.0 \pm 4.4$

0.85

$79.3 \pm 4.3$

0.11

$30.6 \pm 2.4$

0.60

$38.0 \pm 3.3$

0.75

$5.9 \pm 1.5$

0.88

$6.5 \pm 1.6$

0.41

$15.8 \pm 1.2$

0.87

$18.5 \pm 1.3$

0.41

Values are expressed in $\mathrm{mm}$ as mean $\pm \mathrm{SD}$. $P$-values are adjusted for gestational age and gender. Results considered as significant $(\leq 0.05)$ are marked in bold.

INSVM, isolated non-severe ventriculomegaly; W, weeks of gestation; BPD, Biparietal diameter; CC, cranio-caudal diameter

* measurements performed at diagnosis 
Table 3. Sulcal depth for left and right hemisphere of study groups at 26 weeks of gestation

\begin{tabular}{|l|lllll|}
\hline & $\begin{array}{l}\text { Controls } \\
(n=35)\end{array}$ & $\begin{array}{l}\text { All } \\
\text { INSVM } \\
(n=35)\end{array}$ & $\begin{array}{l}\text { Bilateral } \\
\text { INSVM } \\
(n=12)\end{array}$ & $\begin{array}{l}\text { Unilateral } \\
\text { left } \\
\text { INSVM } \\
(n=12)\end{array}$ & $\begin{array}{l}\text { Unilateral } \\
\text { right } \\
\text { INSVM } \\
(n=11)\end{array}$ \\
\hline $\begin{array}{l}\text { Insula } \\
\text { Sylvian fissure }\end{array}$ & $14.2 \pm 1.7$ & $32.0 \pm 2.1$ & $33.1 \pm 1.9$ & $32.2 \pm 2.0$ & $30.8 \pm 2.1$ \\
$\begin{array}{l}\text { Parieto-occipital } \\
\text { sulcus }\end{array}$ & $7.8 \pm 3.0$ & $6.7 \pm 2.2$ & $5.7 \pm 1.5$ & $5.9 \pm 2.0$ & $8.3 \pm 2.2$ \\
$\begin{array}{l}\text { Cingulate sulcus } \\
\text { Calcarine sulcus }\end{array}$ & $1.8 \pm 0.8$ & $1.6 \pm 1.4$ & $1.5 \pm 1.8$ & $1.7 \pm 1.2$ & $1.7 \pm 1.4$ \\
& $9.0 \pm 2.9$ & $7.1 \pm 2.9$ & $6.3 \pm 2.3$ & $6.9 \pm 3.5 *$ & $8.1 \pm 2.8$ \\
\hline
\end{tabular}

\begin{tabular}{|c|c|c|c|c|c|}
\hline $\begin{array}{l}\text { Group } \\
\text { Right } h\end{array}$ & $\begin{array}{l}\text { Controls } \\
(n=35)\end{array}$ & $\begin{array}{l}\text { All } \\
\text { INSVM } \\
(n=35)\end{array}$ & $\begin{array}{l}\text { Bilateral } \\
\text { INSVM } \\
(n=12)\end{array}$ & $\begin{array}{l}\text { Unilateral } \\
\text { left } \\
\text { INSVM } \\
(n=12)\end{array}$ & $\begin{array}{l}\text { Unilateral } \\
\text { right } \\
\text { INSVM } \\
(n=11)\end{array}$ \\
\hline Insu & $30.8 \pm 1.6$ & $31.9 \pm 1.9$ & $33.1 \pm 1.9 *$ & $31.2 \pm 1.4$ & $31.4 \pm 2.0$ \\
\hline Sylvian fisst & $15.8 \pm 2.0$ & $14.6 \pm 2.3$ & $13.7 \pm 1.9$ & $14.8 \pm 2.4$ & $15.3 \pm 2.4$ \\
\hline $\begin{array}{l}\text { Parieto-occipital } \\
\text { sulcus }\end{array}$ & $8.5 \pm 2.5$ & $6.6 \pm 1.9$ & $5.4 \pm 1.1^{*}$ & $8.1 \pm 1.9$ & $6.4 \pm 1.5$ \\
\hline Cingulate sulc & $1.8 \pm 0.8$ & $1.6 \pm 1.3$ & $1.5 \pm 1.7$ & $1.8 \pm 1.3$ & $1.7 \pm 1.0$ \\
\hline Calcarine sulcus & $9.5 \pm 3.0$ & $5.9 \pm 2.7^{*}$ & $5.9 \pm 1.7$ & $6.5 \pm 3.5$ & $5.3 \pm 2.5$ \\
\hline
\end{tabular}

Measurements obtained in $\mathrm{mm}$ were normalized by BPD and multiplied by 100 . Results are expressed as mean \pm SD.

$* p$-values $\leq 0.05$, adjusted by gestational age at examination and gender. INSVM, isolated non-severe ventriculomegaly. 
Table 4. Sulcal depth for left and right hemisphere of study groups at $\mathbf{3 0}$ weeks of gestation

\begin{tabular}{|c|c|c|c|c|c|}
\hline $\begin{array}{l}\text { Group } \\
\text { Left } h\end{array}$ & $\begin{array}{l}\text { Controls } \\
(n=40)\end{array}$ & $\begin{array}{l}\text { All INSVM } \\
(n=40)\end{array}$ & $\begin{array}{c}\text { Bilateral } \\
\text { INSVM } \\
(n=14)\end{array}$ & $\begin{array}{c}\text { Unilateral } \\
\text { left } \\
\text { INSVM } \\
(n=13)\end{array}$ & $\begin{array}{l}\text { Unilateral } \\
\text { right } \\
\text { INSVM } \\
(n=13)\end{array}$ \\
\hline Insu & $30.8 \pm 1.7$ & $\begin{array}{c}31.7 \pm \\
1.8^{*}\end{array}$ & $32.3 \pm 2.0$ & $31.9 \pm 1.7$ & $30.9 \pm 1.4$ \\
\hline Sylvian fissure & $15.8 \pm 2.1$ & $15.6 \pm 2.0$ & $14.2 \pm 2.0$ & $15.9 \pm 1.7$ & $16.6 \pm 1.7$ \\
\hline $\begin{array}{l}\text { Parieto-occipital } \\
\text { sulcus }\end{array}$ & $12.6 \pm 2.4$ & $\begin{array}{c}10.2 \pm \\
2.7^{*}\end{array}$ & $9.3 \pm 2.9 *$ & $10.2 \pm 2.5$ & $11.2 \pm 2.5$ \\
\hline Cingulate sulcus & $6.7 \pm 1.5$ & $5.9 \pm 1.7$ & $5.8 \pm 1.9$ & $5.9 \pm 1.9$ & $5.9 \pm 1.6$ \\
\hline Calcarine sulcus & $12.4 \pm 3.8$ & $10.4 \pm 2.8$ & $10.4 \pm 1.6$ & $8.7 \pm 2.7^{*}$ & $12.0 \pm 3.0$ \\
\hline
\end{tabular}

\begin{tabular}{|c|c|c|c|c|c|}
\hline Group & $\begin{array}{l}\text { Controls } \\
(n=40)\end{array}$ & $\begin{array}{l}\text { All INSVM } \\
(n=40)\end{array}$ & $\begin{array}{c}\text { Bilateral } \\
\text { INSVM } \\
(n=14)\end{array}$ & $\begin{array}{c}\text { Unilateral } \\
\text { left } \\
\text { INSVM } \\
(n=13)\end{array}$ & $\begin{array}{c}\text { Unilateral } \\
\text { right } \\
\text { INSVM } \\
(n=13)\end{array}$ \\
\hline Insula & $31.2 \pm 1.4$ & $31.3 \pm 1.9$ & $31.6 \pm 1.8$ & $31.8 \pm 1.6$ & $30.7 \pm 2.1$ \\
\hline Sylvian fissure & $15.5 \pm 2.1$ & $\begin{array}{c}14.3 \pm \\
2.4^{*}\end{array}$ & $14.3 \pm 2.8$ & $13.8 \pm 2.1^{*}$ & $14.7 \pm 2.1$ \\
\hline $\begin{array}{l}\text { Parieto-occipital } \\
\text { sulcus }\end{array}$ & $13.2 \pm 2.5$ & $\begin{array}{c}11.1 \pm \\
2.2^{*}\end{array}$ & $9.7 \pm 2.7^{*}$ & $11.9 \pm 1.7$ & $11.5 \pm 1.5$ \\
\hline Cingulate sulcus & $6.4 \pm 1.5$ & $6.1 \pm 1.7$ & $5.4 \pm 1.6$ & $5.5 \pm 1.7$ & $6.9 \pm 1.2$ \\
\hline Calcarine & $12.6 \pm 3.9$ & $9.7 \pm 2.4^{*}$ & $10.1 \pm 1.9$ & $9.8 \pm 2.8^{*}$ & $9.1 \pm 2.5^{*}$ \\
\hline
\end{tabular}

Measurements obtained in mm were normalized by BPD and multiplied by 100 . Results are expressed as mean $\pm \mathrm{SD}$.

$* p$-values $\leq 0.05$, adjusted by gestational age at examination and gender. INSVM, isolated non-severe ventriculomegaly. 